\title{
EDITORIAL
}

\section{SIGNS OF COGNITIVE DECLINE IN THE ELDERLY POPULATION}

\author{
K. HUGDAHL
}

Department of Biological and Medical Psychology, University of Bergen, Norway, email: hugdahl@psybp.uib.no, phone +4791181062

Hommet et al. (1) used a variant of the consonant-vowel syllables dichotic listening (DL) procedure, called the "forcedattention" DL paradigm and originally developed by Hugdahl and Andersson in 1986 (2). In brief the paradigm involves two additional conditions to a standard dichotic listening procedure where subjects are explicitly instructed to focus attention and report from either the right or left ear. Instruction subjects to attend to the right ear stimulus in the dichotic situation would enhance the right ear advantage (REA) seen in the default situation with no instruction since attention would help selecting the right ear stimulus. The is not the case in the forced-left condition where the subject has to use top-down attention control to counteract the tendency to report the bottom-up right ear stimulus caused by the lateralization to the left hemisphere of speech sound perception. Considering that the CV-syllables task is quite easy to perform, it should not be more difficult for the patients to understand and perform the task of reporting the left ear stimulus than to perform the task of reporting the right ear stimulus. Thus, the selective left ear reporting failure of the patients was surprising. However, if the "forced-attention" paradigm (3) is thought of as a situation involving a top-down (instruction-driven) modulation of a bottom-up (stimulus-driven) lateralized REA effect (4) it would qualify as a cognitive conflict situation according to the definition given above, and hence the surprise could be explained. Seen as such the failure of the elderly subjects in the study by Hommet et al. to modulate the REA may have been caused by a failure of top-down attention modulation, rather than a failure of bottom-up lateralized perception, as also suggested by the auhtors.

The study by Hommet et al. may therefore contain an important finding that otherwise healthy elderly individuals show signs of a moderate degree of cognitive decline when it comes to the most demanding and highest cognitive functions, namely to resolve a cognitive conflict caused by conflicting response tendencies built in the stimulus situation. This interpretation is evident from the finding by Hommet et al. that there were more intrusions of the right ear stimulus in the forced-left attention condition than left ear intrusions in the forced-right attention condition. If the forced-left attention condition sets up a cognitive conflict between bottom-up and top-down processing demands it would make sense that there should be more right ear intrusions in this condition, because the situation is simply more demanding, requiring additional cognitive resources. In a recent review of the studies in our laboratory in Bergen Norway, we have consistently found that patient groups and extreme stress-situations that from a diagnostic point of view have little in common, like schizophrenia, dementia, PTSD, ADHD, sleep deprivation, all show a decline in the ability to report the left ear stimulus in the forced-left attention siutation. From a neurocognitive point of view this would however be predicted because all these clinical conditions share an impairment of ex executive function and cognitive control, necessary for resolving the cognitive conflict caused by the stimulus and instruction requirements in this situation. We have further documented (5) that the neuronal localization of this effect is in the lateral medial prefrontal cortex, including the dorsal portion of the anterior cingulate, which are areas also found activated in other tasks involving executive functions and cognitive control. An important and often neglected aspect with the forced-attention dichotic listening procedure is that it involves a minimalistics experimental condition between the two attention instruction conditions. In fact the only difference between the two conditions is a single word, namely the direction of attention focus, either "right" or "left", in all other respects the experimental situation is unchanged from one condition to the other. This may be important when studying higher cognitive functions, because often the tests used in traditional neuropsychological test batteries also differ in level of difficulty and requirements for general understanding of the task to be performed. This way be a confounding factor when studying impaired clinical and para-clinical groups. The dichotic listening task is also very easy to perform and should be easily tolerated by most patients.

Thus, Hommet et al. are to be congratulated with an interesting and important study, showing early sign of decline in using cognitive control strategies to resolve a cognitive conflict caused the opposing response tendencies of the nature of the stimulus and the nature of the attention instruction in the force-left condition. As such the study may add to a growing amount of evidence showing subtle impairments in old age at a sub-clinical level.

\section{References}

1. Hommet, C., Mondon, K., Berrut, G., Gouyer, Y., Isingrini, M., Constans, T., Belzung, C. Central auditory processing in aging : The dichotic listening paradigm. J Nutr Health Aging. 2010;14(9):751-756.

2. Hugdahl, K., Westerhausen, R., Alho, K., Medvedev, S., Laine, M., Hämäläinen, H (2009). Attention and cognitive control: Unfolding the dichotic listening story. Scandinavian Journal of Psychology, 50, 11-22.

3. Hugdahl, K., Andersson, L. (1986). The "forced-attention paradigm" in dichotic listening to CV-syllables: A comparison between adults and children. Cortex, 22, 417-432.

4. Hiscock, M., Inch, R., Kinsbourne, M. (1999). Allocation of attention in dichotic listening: Differential effects on detection and localization of signals. Neuropsychology, 13, 404-414.

5. Thomsen, T., Rimol, L.M., Ersland, L. Hugdahl, K. (2004). Dichotic listening reveals functional specificity in prefrontal cortex : An fMRI study. Neuroimage, 21, 211-218. 